\title{
Stable Increased Formulation Atomisation Using a Multi-Tip Nozzle Device
}

Rita Haj-Ahmad ${ }^{1}$, Manoochehr Rasekh ${ }^{1}$, Kazem Nazari ${ }^{1}$, Ekhoerose V. Onaiwu ${ }^{1}$, Bushra Yousef $^{1}$, Stuart Morgan ${ }^{2}$, David Evans ${ }^{2}$, Ming-Wei Chang ${ }^{3,4}$, John Hall $^{5}$, Chris Samwell ${ }^{5}$ and Zeeshan Ahmad ${ }^{1 *}$

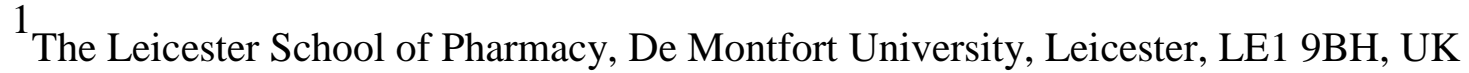

${ }^{2}$ Genvolt, New Road, Bridgnorth, Shropshire, WV16 6NN, UK

${ }^{3}$ College of Biomedical Engineering and Instrument Science, Zhejiang University, Hangzhou 310027, China

${ }^{4}$ Zhejiang Provincial Key Laboratory of Cardio-Cerebral Vascular Detection Technology and Medicinal Effectiveness Appraisal, Zhejiang University, Hangzhou 310027, China

${ }^{5}$ BlueFrog Design, 19 St. Margarets Street, Leicester, LE1 3EB, UK

*Corresponding Author:

Prof. Zeeshan Ahmad

Email: zahmad@dmu.ac.uk

Tel: $+44(0) 1162506455$ 


\begin{abstract}
Electrohydrodynamic atomisation (EHDA) is an emerging technique for the production of micron and nano-scaled particles. The process often involves Taylor cone enablement, which results in a fine spray yielding formulated droplets, which then undergo drying during deposition. In this work novel multi-tip emiiter (MTE) devices were designed, engineered and utilised for potential upscaled EHDA, by comparison with a conventional single needle system. To demonstrate this, the active ketoprofen (KETO) was formulated using polyvinylpyrrolidone (PVP) polymer as the matrix material. Here, PVP polymer $(5 \% \mathrm{w} / \mathrm{v})$ solution was prepared using ethanol and distilled water (80:20) as the vehicle. KETO was incorporated as 5\% w/w of PVP. Physical properties of resulting solutions (viscosity, electrical conductivity, density and surface tension) were obtained. Formulations were electrosprayed through both single and novel MTEs under EHDA conditions at various flow rates $(5-300 \mu \mathrm{l} / \mathrm{min})$ and applied voltages $(0-30 \mathrm{kV})$. The atomization process using MTEs and single nozzle was monitored at using various process parameters via a digital optical camera. Resulting particles were collected $200 \mathrm{~mm}$ below processing heads and were analyzed using differential scanning calorimetry (DSC), thermal gravimetric analysis (TGA), X-ray diffraction (XRD) and scanning electron microscopy (SEM). Digital recordings confirmed stable MTE jetting at higher flow rates. Electron micrographs confirmed particle size variation arising due to nozzle head design and evidenced stable jetting derived greater near uniform particles. DSC, XRD and TGA confirm KETO molecules were encapsulated and dispersed into PVP polymer particles. In conclusion, novel MTE devices enabled stable atomisation even at higher flow rates when compared to the conventional single needle device. This indicates an exciting approach for scaling-up (EHDA) in contrast to current efforts focusing on multiple nozzle and pore based processing outlets.
\end{abstract}

Keywords: Multi-tip emitter (MTE), electrospraying, nanoparticles, microparticles, jetting, electrohydrodynamic, encapsulation 


\section{Introduction}

The last decade has witnessed several engineering advances and developments for nano and micrometer scaled particle engineering using methods such as spray drying, emulsification-diffusion, supercritical fluids and electrohydrodynamic atomisation (EHDA). The latter is perhaps the most recent technological development and has attracted significant interest due to several advantages over existing manufacturing and process technologies (e.g. one-step process, simultaneous hydrophilic and hydrophobic active incorporation, ease in scaling particle size and facile approach to alter general morphology e.g. fiber to particle and vice versa) (1).

The underlying principle surrounding EHDA is based on the application of an electric field to a flowing and electrically conducting liquid, which in many instances is a pharmaceutical formulation. This, respectively, is what identifies the 'Hydro' and 'Dynamic' in the term EHDA. The meniscus generated at the nozzle exit becomes highly charged and a stable cone jet is then formed. The applied electric field between the tip of the nozzle and the collecting substrate drives the process further, overcoming surface tension, yielding a break-up of the jet causing fine droplets to form. Upon drying these droplets give rise to medicated particles based on the initial formulation (2). Primarily, highly charged micro-meter droplets are formed which then split into finer particles due to electrostatic repulsion experienced within the electric field. The mode (and stability) of spraying is considered the most significant feature within EHDA processes (2).

Several physical properties of the formulation (surface tension, density, viscosity and electrical conductivity) impact the ease and viability of atomisation. Process parameters such as flow rate, applied voltage, distance between nozzle outlet and the collecting platform; dielectric strength of the ambient atmosphere and nozzle configuration all have an influence on the architecture and morphology of the final structures (3). To date, several EHDA device configurations have been developed. Nozzle designs have varied from simple single needles (various diameters) to more complex and multiplexed systems. The production of nanoparticles via single needle electrospraying, however, entails the application of low flow rates at elevated electric fields. Bocanegra et al., 2005 reported that in order to develop a $1 \mu \mathrm{m}$ droplet, the electric conductivity and flow rate of deployed liquid should be $1 \mathrm{mS} / \mathrm{m}$ and $10 \mu \mathrm{l} / \mathrm{hr}(0.16 \mu \mathrm{l} / \mathrm{min})(4)$. The use of such low formulation flow rates is a major limitation for single needle electrospraying systems for 
industrial applications, unless several nozzles are used in parallel during the process. The requirement of appreciable commercial scale production in shorter timeframes is a key facet for most pharmaceutical and biomedical industries. Numerous electrospray configurations have been developed aiming to scale-up or demonstrate increased output rates (4-6). For instance, a linear array of parallel needles (in square arrangement) has been developed by Rulison and Flagan (1993) (5). In 1999, Almekinders and Jones arranged 24 spraying nozzles in a $15 \mathrm{~cm}$ width standard EHD atomizer. Standard oil was used and applied voltages of $35 \mathrm{kV}$ and flow rates between 1000-3333 $\mu$ l.min-1 were deployed. Resulting droplet diameters were in the range 80-400 $\mu \mathrm{m}$ (6) which are considered coarse compared to particles produced using a single needle device. Bocanegra et al., (2005) developed an array of 37 orifices (instead of nozzles) pierced in dielectric constituents with hydrophobic surfaces in a hexagonal pattern $(\sim 6 \mathrm{~mm})$ with an extractor which possessed the same number of orifices and pattern design to the emitter surface. Up to 115 cone jets were reported per square centimetre; whilst deploying a flow rate of $83 \mu 1 . \mathrm{min}^{-1}$. Each orifice displayed independent single cone-jet formation due to segregation from neighbouring outlets (4). Yang et al., 2010 developed a hexagonal-distributed multi-needle electrospinning configuration possessing a shield ring design in which each set of 3 needles (of the spinneret) were placed as symmetrical triangles. Near uniform and smaller nanofibers were produced using more needles on the spinneret (7).

The flow rate of liquid from single needle setup is typically in the range $\sim 16-20 \mu$ l.min ${ }^{-1}$ which allows the production of homogenous surface structures. The use of very low flow rates may result in reduced dissipation from the nozzle tip and yield irregular structure formation. In contrast, the deployment of elevated flow rates can result in very large droplets with incomplete solvent evaporation. There exists a direct relationship between particle size and flow rate for a given liquid (formulation). For example, Jafari-Nodoushan et al., 2015 reported that by increasing the flow rate from 33.3 to $83.3 \mathrm{ml} / \mathrm{hr}$, the average particle diameter increases from 14.6 to $28 \mu \mathrm{m}(8)$.

In this work, novel EHDA nozzle and proof-of-concept design is developed which provides multiple flow outlets for a single infused electrospraying (or electrospinning) formulation. The system consists of multi-tip emitters (MTEs) through which an API-polymer solution is imposed. The tips are intended to facilitate charge distribution (via spatial and angled metallic projections) throughout the cone and jet, while the voids enable enhanced formulation media inflow. 
Conducting tips (from MTE heads) differed in their length indicating the impact of electric field and electrical conductivity at the cone even at elevated flow rates. Using hydrophobic antiinflammatory drug 'ketoprofen (KETO)' as the active pharmaceutical ingredient (API); particle size and distribution, drug encapsulation, state and release were explored. The findings indicate an alternative and exciting route to scale-up through a directional concept change albeit still incorporating all the benefits of EHDA processes.

\section{Materials and Methods}

\section{Materials}

Crystalline Ketoprofen (KETO), polyvinylpyrrolidone (PVP, high purity grade) $\left(4.4 \times 10^{4} \mathrm{~g} / \mathrm{mol}\right)$ and phosphate buffer saline (PBS) were obtained from Sigma-Aldrich (Gillingham, UK). Ethanol (HPLC grade) was purchased from Fisher Scientific (Loughborough, UK). Purified water was obtained in house.

\section{Methods}

\section{Formulation Preparation}

A total volume of $75 \mathrm{~mL}(5 \% \mathrm{w} / \mathrm{v})$ PVP solution was prepared using ethanol and distilled water (80:20, respectively) as the vehicle. PVP was dissolved in the co-solvent system through mechanical stirring for 60 minutes (Jenway, Staffordshire, UK). The resulting solution was sealed with parafilm to avoid solvent evaporation. KETO (5\% w/w of PVP) was added to the solution under mechanical stirring for a further 60 minutes to ensure complete dissolution.

\section{Physical properties of Solvents and Formulations}

The viscosity, electric conductivity, density and surface tension of all solvents and formulations were determined. Viscosity was measured at ambient temperature $\left(23^{\circ} \mathrm{C}\right)$ using a SV-10 Sinewave Vibro Viscometer (A\&D, Oxfordshire, UK). A FG3-FIVEGOTM Conductivity Meter (Mettler Toledo, Leicester, UK) was used to measure the electrical conductivity of all formulations and solvent systems. Here, the probe was calibrated with a standard $12.88 \mathrm{mS} / \mathrm{cm}$ solution prior to use. Density values were obtained using standard $50 \mathrm{~mL}$ pyncnometers (VWR, Leicestershire, 
UK). Surface tension was estimated using 0/80256E Balance Tensiometer (White Elec. Inst. Co., Ltd., Worcestershire, UK) using the DuNuoy platinum ring technique. All measurements were performed in triplicate.

\section{Electrohydrodynamic Atomisation Using MTE and Single Nozzle System}

PVP-KETO formulation was infused into a single stainless steel needle (OD 3mm) and MTE spraying head systems (possessing 600 and $1200 \mu \mathrm{m}$, tip lengths, termed $600 \mathrm{MN}$ and $1200 \mathrm{MN}$ hereafter) at following flow rates: 5, 10, 100 and $300 \mu 1$. min $^{-1}$. The infusion rate was controlled using a Harvard Apparatus 11-Elite syringe pump (Harvard Apparatus, Edenbridge, UK). An electric field $(0-30 \mathrm{kV})$ was supplied via a high voltage power supply (Genvolt, Model 7303). Particles were collected at a working distance of $200 \mathrm{~mm}$ below device exit (designed and engineered at BlueFrog Design and De Montfort University) on glass slide substrates (microscopic slides, WWR, Leicester, UK). An image of the EHDA system set-up is shown Figure 1 and Figure $\mathbf{2}$ shows components and configuration of MTEs. Figure 3 shows key components of conventional nozzle heads and selected recent atomizer designs and current MTE technology.

\section{Digital and Microscopic Imaging}

Jetting images from spraying heads were captured using a GXCAM Hichrome-Met-UK. Scanning electron microscopy (SEM) was performed using SEM Carl Zeiss EVO ${ }^{\circledR}$ HD15 (Carl, Zeiss, Oberkochen, Germany). Particles were mounted onto the sample holder (aluminium stub) using double-sided tape. Particles were gold coated using a sputter coater S150B (Edwards, Crawley, West Sussex, UK) to prevent overcharging. Particle diameter and distribution were examined via SEM image analysis program Image Tool in which mean particle diameter and size distribution were defined for 100 random measurements as a representation of particle morphology. Contact angle behaviour on MTE was measured using "Theta light" (Biolin Scientific Attension, Stockholm, Sweden). A droplet of water was placed onto the surface of inverted MTEs which was assessed using a video monitor. 


\section{Differential scanning calorimetry (DSC)}

DSC analysis of formulated and atomised particles was performed using a differential scanning calorimeter (PerkinElmer Jade DSC (PerkinElmer Ltd., Shelton, CT, USA)). Samples (3.5 - 4.2 mg) were sealed into aluminium pans (Perkin Elmer) and loaded into sample cells under nitrogen. Samples were scanned in the temperature range $20-200{ }^{\circ} \mathrm{C}$ at an underlying heating rate of $10^{\circ} \mathrm{C} / \mathrm{min}$ (nitrogen purge of $70 \mathrm{~mL} / \mathrm{min}$ ).

\section{Thermogravimetric analysis (TGA)}

Thermal behaviour of formulated and atomised particles was evaluated using a thermal gravimetric analyser TGA (Perkin Elmer Pyris 1 TGA). Each sample (7-10 mg) was placed in an aluminium pan (TGA, Perkin Elmer) and heated at a temperature range of 20 to $800^{\circ} \mathrm{C}$ in nitrogen atmosphere, using a heating rate of $10{ }^{\circ} \mathrm{C} / \mathrm{min}$.

\section{Fourier transform infrared spectroscopy (FTIR)}

The chemical structure of raw materials and electrosprayed particles was investigated by Fourier Transform infrared spectroscopy (FT-IR Platinum-ATR fitted with Bruker Alpha Opus 27 FT-IR). Infra-red spectra were obtained in the range $4000-400 \mathrm{~cm}^{-1}$ at a resolution of $4 \mathrm{~cm}^{-1}$ for each run at the ambient temperature.

\section{X-ray diffraction studies (XRD)}

Crystallinity of resulting particulate samples was evaluated using XRD analysis performed on a Bruker D2- Phaser diffractometer, operating at $40 \mathrm{kV}$ and $40 \mathrm{~mA}$, using $\mathrm{Cu} \mathrm{Ka}$, at a scanning rate of $0.35 \mathrm{sec} / \mathrm{step}$. Spectra were collected in $2 \theta$ from -3 to $+160^{\circ}$.

\section{Encapsulation Efficiency and In vitro Release}

Accurately, $1 \mathrm{mg}$ of each particulate material was weighed and dissolved in $3 \mathrm{~mL}$ of PBS in capped glass vials under magnetic stirring for $10 \mathrm{~min}$. Quantification of KETO was performed using UVVis analysis at $260 \mathrm{~nm}$ (UV-Vis spectrophotometer, Shimadzu, UVmini-1240). Experiments were run in triplicate. Drug content was calculated using Equation 1: 
(Eq. 1) Encapsulation Efficiency (\%) $=($ W KETO-PRT/ W KETO-USED) $x 100$

Where W KETO-PRT is the weight of KETO in particles and W KETO-USED is the theoretical quantity of KETO used in formulation.

The rate of KETO release was determined in PBS (pH 7.4). The release study was performed in capped glass vials placed in a shaking water bath at $37{ }^{\circ} \mathrm{C} .5 \mathrm{mg}$ of electrosprayed samples (obtained using flow rates of 10 and $300 \mu 1 . \mathrm{min}^{-1}$ ) were added to $20 \mathrm{~mL}$ of PBS buffer solution (pH 7.4). At pre-set time intervals, $1 \mathrm{~mL}$ of each sample was withdrawn and replaced with an equal volume of fresh PBS solution. Samples were assessed in triplicate using single beam scanning UV/Visible spectrophotometry (Model M501, Camspec Ltd., UK) at $260 \mathrm{~nm}$. All measurements were performed in triplicate. The calibration curve of KETO (in PBS pH 7.4) displayed good linearity $\left(r^{2}=0.999\right)$.

\section{Statistical analysis}

Levene's test was applied to assess the uniformity of variances. Results were assessed by one-way ANOVA test followed by SPSS software (Version 15.0, SPSS Incorporation, USA). $p$ value less than 0.05 was considered to be significantly different.

\section{Results and Discussion}

\section{Solution characterisation}

Formulation physical properties play an important role in predicting and explaining electrospraying jetting processes. Table 1 shows solution and formulation characteristics namely; surface tension, density, electrical conductivity and viscosity. Surface tension is a limiting step in the electrospraying process. It has been reported that the surface tension of a liquid should be less than $50 \mathrm{MTE} / \mathrm{m}$ to be atomised under the influence of electrical forces (9). In this study, the surface tension of all media were in the range $0.032-0.038 \mathrm{MTE} / \mathrm{m}$ (less than 50MTE/m), in which there was no influence of drug or polymer.

Electrical conductivity refers to the ability of materials to accommodate the transport of electric 
charges (10). Liquids possessing low electrical conductivities $\left(<10^{-6} \mu \mathrm{S} . \mathrm{cm}^{-1}\right)$ cannot be used for electrospraying alone (e.g. heptane) (11). The electric conductivity of ethanol has a higher value $\left(0.127 \mu \mathrm{S} . \mathrm{cm}^{-1}\right)$ which increased to 0.605 upon addition of $(20 \% \mathrm{v} / \mathrm{v})$ water, and significantly increased by adding drug, polymer or both together.

Liquid viscosity impacts the size of final structure as this property significantly affects the jet break-up process (11). From the data, an increase in viscosity is observed upon addition of water, PVP and KETO to pure ethanol solution. Liquid density is related to viscous forces which play a role in transmission of electric charge through the jet diameter. Density also has an effect on particle size; as increasing density reduces the flow rate required for stable Taylor cone formation (11). Data obtained for density shows no significant effect in relation to polymer and API addition.

MTE design enabled enhanced flow rate input; better suited for large-scale production potential. This is timely since most literature involve or details flow rate usage $<20 \mu 1 . m^{-1}$. Once formulation flows through a capillary under the influence of an applied voltage, various jetting modes are encountered through incremental changes to process parameters; mainly applied voltage for a given flow rate. However, sprayed particles at this stage will not be near uniform in size. A crucial step in the electrospraying process is to achieve the Taylor cone that leads to the production of near uniform micron or sub-micron particles (12). The Taylor cone develops in the following sequence: at zero applied voltage, a hemispherical droplet evolves at the tip of the nozzle as a result of interaction involving liquid surface tension and gravity. Once a voltage is applied, a conical shape is formed; which is influenced by polarization from the air/liquid interface. When an equilibrium between free liquid surface and sufficient electric field is reached at the tip of the nozzle, the Taylor cone is achieved $(5,13,14)$. Contact angle measurements on MTEs (Fig. 4) indicate pipetted liquid droplets are able to flow through template voids within $0.2 \mathrm{~s}$. This indicates clearly that novel MTE systems do not cause liquid/formulation assembly at the outlet interface which may arise due to trapped bubbles or air pockets.

In the current study, two novel MTE configurations (600 and $1200 \mathrm{MN}$ ) were used in addition to a conventional single needle-nozzle EHDA processing system. Various flow rates (5, 10, 100 and $300 \mu 1 . \mathrm{min}^{-1}$ ) were selected to investigate jetting stability from MTEs. An electric field was 
applied (range $\sim 5-29 \mathrm{kV}$ ) until a stable cone jet was observed. Fig 5 shows jetting images of PVP-KETO formulations using single needle, 600 and $1200 \mathrm{MNs}$ processing heads. Observations of the single needle system show stable cone-jets which were easily obtained by applying low flow rates (e.g. 5 and $10 \mu 1 . \mathrm{min}^{-1}$ ). However, increasing the flow rate to $100 \mu 1 . \mathrm{min}^{-1}$ had an adverse effect on formulation jetting modes from the single nozzle system. Multi-jets were observed, and relatively large droplets were produced. Utilising flow rates between 100-300 $\mu 1 . \mathrm{min}^{-1}$ leads to increased process instability as shown in Fig 5 and various jetting attributes can be seen in Table 2. Conventionally, low flow rates are ideal for near-uniform micron scaled particle engineering using single nozzle systems (12) and this is evident in electron micrographs shown in Fig 6; as increasing the flow rate results in larger particles (8). However, the use of low flow rate requires applying a low voltage which usually leads to the production of smaller particles (15). This affects the quantity of generated particles thus limiting its large-scale potential in pharmaceutical and biomedical industries.

For both MTE configurations (600 and $1200 \mathrm{MN}$ ) cone-jet stability at low flow rates (such as 5 $\mu 1$. min $^{-1}$ ) is compromised and greater formulation flow rates are required (e.g. $\geq 10 \mu 1 . m^{-1}$ ). Spherical and smooth surfaced particles (Figure 6) with narrow size distribution were obtained using $10 \mu 1 . \mathrm{min}^{-1}$ (all particle were less than $1 \mu \mathrm{m}$ in size, as shown in Figure 7). Furthermore, MTE systems required greater voltage application (e.g. $\geq 20 \mathrm{kV}$ ) in order to achieve stable cone jets and produce particles when deploying high flow rates (e.g. $100-150 \mu 1 . \mathrm{min}^{-1}$ ) were deployed. This is due to the large number of voids within MTEs; which required greater electric field strength and charge to deform the entire droplets into a cone-jet (16); which subsequently breaksup to yield small particles (10). When increasing the flow rate to $300 \mu 1 . \mathrm{min}^{-1}$, both MTEs formed a Taylor cone. The meniscus at the apex of the $600 \mathrm{MN}$ system overflowed intermittently and spread onto the surrounding surface resulting in particles with broader size distribution (Figure 7). This indicates a modulated higher voltage may be needed to electrify the solution and control the spraying process.

The mean particle diameter of electrosprayed particles was found to vary depending on the nozzle system. Fig. 7 illustrates the size distribution (histogram) of at least 100 particles from each 
atomizing system. It was observed that particles produced using $1200 \mathrm{MN}$ (at a high flow rate of $100 \mu 1$. min $^{-1}$ ) were characterised by a narrow size distribution when compared with the single tip nozzle. Here, $\sim 52 \%, \sim 85 \%$ and $\sim 90 \%$ of particles utilised using single, 600 and $1200 \mathrm{MN}$ systems where less than $1 \mu \mathrm{m}$ in size, respectively. Electron micrographs showing electrosprayed particles prepared using MTEs (between 100 and $300 \mu 1 . \mathrm{min}^{-1}$ ) were near monodisperse and spherical in nature indicating their potential to efficiently fabricate particles as proficiently as single needle configurations albeit at increased output rates.

\section{Encapsulation efficiency and release study}

The impact of flow rate on the KETO-encapsulation efficiency and KETO-release rate was studied. Table 3 shows encapsulation efficiency of particles fabricated using single needle, 600 and $1200 \mathrm{MN}$ at two selected flow rates; 10 and $300 \mu 1 . \mathrm{min}^{-1}$. The encapsulation efficiency of KETO at $10 \mu 1 . \mathrm{min}^{-1}$ was found to be in the range of $79-88 \%$. The values were significantly higher than those reported for particles produced at higher flow rates $\left(300 \mu 1 . \mathrm{min}^{-1}\right)$; especially for particles produced using single needle systems. KETO release from polymeric particles is shown in Fig 8. In vitro KETO release from electrosprayed particles produced at $10 \mu 1 . \mathrm{min}^{-1}$ (Figure 8A) exhibit rapid release due to excellent wettability of the polymer matrix (PVP). A sustained release period is also apparent which is due to further dissolution of KETO, since KETO co-exists within polymeric particles in an amorphous and homogeneous state (17). There was no significant difference between KETO release from electrosprayed particles prepared at $10 \mu 1$. min $^{-1}$ when using single needle, 600 or 1200 MNs.

However, the case was different after increasing the flow rate to $300 \mu 1 . \mathrm{min}^{-1}$ as significant $(\mathrm{p}$ $<0.05$ ) differences were observed for KETO release from single needle and MTEs (Fig 8B). $\sim 100 \%$ of the drug was released in the first hour from particles prepared using the single needle while a sustained release profile was obtained for particles prepared using MTEs (600 and 1200 MNs). Particle size and encapsulation efficiency are known to have a significant influence on the drug-release pattern (18). This could be due to the low entrapment efficiency of the drug $(\sim 32.14$ 
$\pm 5.3 \%$ ) and the various particle sizes. Large error bars can be seen (Fig 8B) over the release profile of KETO-polymeric particles prepared using the single needle system which is a result of the broad particle size distribution indicating varied drug encapsulation. However, 45\% of the drug was released within the first hour and continued with a sustained release profile over the eight hour study period.

\section{Differential scanning calorimetry (DSC)}

Fig 9A shows DSC thermograms of pristine materials (PVP and KETO) in addition to their corresponding physical mixture and electrosprayed particles (MTEs prepared samples). Thermograms of pure PVP shows an endothermic peak in the range $\sim 171-182^{\circ} \mathrm{C}$, demonstrating the hygroscopic nature of the polymer (19). For PVP polymers, the glass transition temperature depends on their moisture content and molecular weight $(19,20)$. Pure KETO exhibits a single

endothermic peak ranging from 89 to $109^{\circ} \mathrm{C}$, with a melting temperature $\left(\mathrm{T}_{\mathrm{m}}\right)$ of KETO at 98.3 ${ }^{\circ}$. The physical mixture of PVP-KETO shows a broad endothermic peak with lower intensity and lower melting temperature ranging from 60 to $171^{\circ} \mathrm{C}$, related to melting temperatures of KETO and PVP, identifying the presence of crystalline KETO. The melting peak of KETO could not be observed as it overlaps with the broad endothermic peak in addition to the low loading of KETO in the physical mixture (21). The low intensity and melting peak of the physical mixture is related to the dilution effect of PVP in the mixture (22) and possible heat-induced interactions between KETO and PVP $(19,23)$. A clear difference was observed between the physical mixture and electrosprayed particles. In the physical mixture, KETO is not dispersed efficiently within the polymeric matrix mixture; accordingly, the melting point of the mixture is closer to $\mathrm{T}_{\mathrm{m}}$ of the drug (24). In the DSC thermogram of electrosprayed PVP-KETO particles (prepared using MTEs), no endothermic peaks of KETO are observed, while one endothermic peak ranging from 143-182 ${ }^{\circ} \mathrm{C}$ with a transition temperature (at $146.8 \pm 0.2^{\circ} \mathrm{C}$ ) is detected. This indicates a significant reduction in KETO crystallinity; in addition to the inhibitory effect of PVP on KETO crystallisation (19). This leads to the transformation of KETO into amorphous form $(25,26)$ and/or KETO being molecularly dispersed within the polymeric particles and encapsulated in an amorphous state 
$(24,17)$, regardless of the tip system (600 or $1200 \mathrm{MN})$. The advantage of this transformation from crystalline to amorphous state is that amorphous materials have excess free energy, entropy, enthalpy, higher molecular mobility, lower density and higher specific volume in comparison with crystalline materials. Amorphous materials can be visualised as supercooled liquids rather than solids due to the flexible mechanical properties of amorphous materials $(27,28)$. Moreover, amorphous materials are often characterised by better dissolution profiles as less activation energy is required to promote dissolution when compared to ordered structure of crystals (29). Accordingly, this improves solubility of poorly soluble APIs several folds $(28,30)$ and enhances their bioavailability $(9,31,32)$.

\section{Thermal gravimetric analysis (TGA)}

TGA was used to investigate thermal stability and degradation of electrosprayed particles prepared using MTEs. Fig. 9B shows TGA thermograms of pure KETO, PVP and electrosprayed PVP/KETO particles. Pure PVP and electrosprayed PVP-KETO particles exhibit two degradation steps; the first step is below $200^{\circ} \mathrm{C}$, and is due to evaporation of residual solvent or any surface adsorbed moisture on particles. The second major step is (from 350-480 $\mathrm{C}$ ) due to decomposition of polymer. The second step for PVP-KETO particles is larger than that observed for pure PVP. This also confirms KETO loading in electrosprayed particles using MTEs. As in previous studies, it has been reported that any shift (24) or increase (33) in degradation temperature (relating to weight loss) also indicates API encapsulation within the polymeric structure.

\section{Fourier transform infrared spectroscopy (FTIR)}

Changes in KETO structure and any possible interactions between KETO and PVP were investigated using FT-IR. Fig 10A shows FT-IR spectra (in the range $4000-400 \mathrm{~cm}^{-1}$ ) of pristine materials and electrosprayed particles prepared using MTEs. The FT-IR spectra of PVP shows two sharp peak at $2960 \mathrm{~cm}^{-1}$ (C-H stretch) and $1644 \mathrm{~cm}^{-1}(\mathrm{C}=\mathrm{O})$. Two well defined peaks of crystalline KETO were visible in the $v(\mathrm{C}=\mathrm{O})$ stretching region: one at $1694 \mathrm{~cm}^{-1}$ which indicates the stretching vibration of carbonyl group in the dimeric carboxylic acid as KETO molecules bond together (forming dimers). The second peak was observed at $1654 \mathrm{~cm}^{-1}$ which represents 
stretching vibration of the carbonyl bond $(17,34)$. In addition, IR spectrum in the region of 860 $640 \mathrm{~cm}^{-1}$ indicates presence of aromatic rings (35). These peaks were also observed in physical mixture spectrum; in addition a small peak was observed at $1689 \mathrm{~cm}^{-1}$. A portion of KETO is still available in the crystalline state which is evident from stretching of dimeric $\operatorname{KETO}(34,36)$. PVP can form hydrogen bonds with KETO; as the polymer has two proton acceptor groups $(\mathrm{C}=\mathrm{O}$ and $=\mathrm{N}^{-}$) in its chemical structure. However, the nitrogen atom cannot involve in any intermolecular interactions due to the steric hindrance, that gave the advantage to the carbonyl group for hydrogen bonding with the carbonyl group of drug $(19,25)$. KETO peaks were not visible in the FTIR spectra of the electrosprayed PVP-KETO using (600 and 1200) MTE, indicating the interruption/breakage of the interactions between KETO molecules and the formation of hydrogen bonding between the hydroxyl group of KETO and the carbonyl group of PVP.

\section{X-ray diffraction (XRD)}

The physical state of KETO in the electrosprayed samples prepared using MTEs in comparison with the physical mixture was investigated using XRD.

XRD was used to acquire information about molecular arrangement within the crystalline form of particles. Fig 10B shows the X-ray diffraction patterns of the KETO and PVP, as raw materials, their physical mixture and the electrosprayed particles using MTEs. X-ray pattern demonstrates that the KETO exhibited highly crystalline characteristics as several distinct sharp diffraction peaks can be observed in the KETO diffractogram. The PVP spectrum shows a diffused background pattern with two diffraction halos, which indicates that PVP is amorphous. Sharp peaks from KETO can still be observed with dramatic decline of intensity in the physical mixture, indicating that KETO is present in crystalline form in the mixture, albeit in reduced quantities. According to the literature, by mixing and groundling a crystalline drug with a polymeric excipient, roughly $50 \%$ of the drug becomes amorphous (37) due to the strong interactions between the drug and polymer (34). In this instance, PVP chains separate KETO molecules decreasing their mobility (37). The sharp diffraction peaks of KETO disappeared in electrosprayed particles concluding that all KETO molecules are present in amorphous state using MTEs.

FTIR spectroscopy and XRD results indicate that all electrosprayed PVP-KETO samples using 
MTEs are available in amorphous solid dispersions, while the physical mixture has a portion of the drug in crystalline form.

\section{Conclusion}

In conclusion, MTEs enable stable Taylor cones at elevated flow rates when compared to traditional single needle systems. This, in turn, results in greater medicated particle production rates. Particle size was shown to be more uniform when prepared using MTEs in comparison with single needle systems. Overall, MTEs provide an alternative approach for stable increasing particle production for EHDA processes.

\section{Acknowledgments}

The authors would like to thank the EPSRC EHDA Network and The Royal Society(Industrial Fellowship) for their support.

\section{List of Figures}

Fig 1. Experiment setup and method for EHDA

Fig 2. Schematic head design. A and B are microneedles with different size. $\mathrm{C}$ is a single needle. $\mathrm{D}$ is the design of microneedle. $\mathrm{E}$ and $\mathrm{F}$ are microneedles image. $\mathrm{G}$ and $\mathrm{H}$ are SEM image of microneedles.

Fig 3. Key components of conventional nozzle heads and selected recent atomizer designs and current MTE technology

Fig 4. Contact angle measurements.

Fig 5. Jetting images from various processing heads at different flow rates $(5,10,100$ and 300 $\mu 1$. min $^{-1}$ )

Fig 6. Electron micrographs of particles produced using single needle, 600 and 1200 MN MTEs at different flow rates: 5, 10, 100 and $300 \mu 1 . \mathrm{min}^{-1}$. 
Fig 7. Diameter size distribution histogram of particles produced at A. $10 \mu 1 . \min ^{-1}$, B. 100 $\mu 1 . \min ^{-1}$ and C. $300 \mu 1$. min $^{-1}$ (mean \pm S.D., $n=100$ ).

Fig 8. Release profiles of particles produced using single needle, 600 and 1200 MN MTEs at different flow rates: A. $10 \mu 1 . \mathrm{min}^{-1}$ and B. $300 \mu 1$. min $^{-1}$ (mean \pm S.D., $\mathrm{n}=3$ ).

Fig 9. A. DSC thermograms of KETO, PVP, PVP/KETO physical mixture (PM) and PVP/KETO particles produced using $1200 \mathrm{MN}$ MTE at flow rate of $10 \mu 1 . \mathrm{min}^{-1}$. B. TGA curves of KETO, PVP, PVP/KETO particles produced using $1200 \mathrm{MN}$ MTE at flow rate of $10 \mu 1 . \mathrm{min}^{-1}$.

Fig 10. A. FT-IR and B. XRD spectra of KETO, PVP, PVP/KETO physical mixture and PVP/KETO particles produced using $1200 \mathrm{MN} \mathrm{MTE} \mathrm{at} \mathrm{flow} \mathrm{rate} \mathrm{of} 10 \mu 1 . \mathrm{min}^{-1}$.

\section{List of Tables}

Table 1. Characterisation of solutions

Table 2. Spray characteristics of 600 and 1200 MN MTEs at 100 and $300 \mu 1 . \mathrm{min}^{-1}$

Table 3. Encapsulation of particles produced using single needle, 600 and 1200 MTE using two different flow rates $\left(10\right.$ and $\left.300 \mu 1 . \min ^{-1}\right)(n=3)$

\section{Conflict of interest}

All authors report no conflict of interest for this research. 


\section{References}

(1) Mehta P, Haj-Ahmad R, Rasekh M, Arshad MS, Smith A, van der Merwe SM, Li X, Chang MW, Ahmad Z. Pharmaceutical and biomaterial engineering via electrohydrodynamic atomization technologies. Drug Discov. Today 2017, 22, 157-165

(2) Jaworek A. Micro- and nanoparticle production by electrospraying. Powder Technol 2007 7/10;176(1):18-35.

(3) Jafari-Nodoushan M, Mobedi H, Barzin J. Encapsulation via Electrohydrodynamic Atomization Spray Technology (Electrospraying). In: Munaya Mishra, editor. Handbook of Encapsulation and Controlled Release: CRS press; 2016. p. 411-437.

(4) Bocanegra R, Galán D, Márquez M, Loscertales IG, Barrero A. Multiple electrosprays emitted from an array of holes. J Aerosol Sci 2005 12;36(12):1387-1399.

(5) Rulison AJ, Flagan RC. Scale-up of electrospray atomization using linear arrays of Taylor cones. Rev Sci Instrum 1993;64(3):683-686.

(6) Almekinders J, Jones C. Multiple jet electrohydrodynamic spraying and applications. J Aerosol Sci 1999;30(7):969-971.

(7) Yang Y, Jia Z, Li Q, Hou L, Liu J, Wang L, et al. A Shield Ring Enhanced Equilateral Hexagon Distributed Multi-needle Electrospinning Spinneret. IEEE Trans Dielectr Electr Insul 2010 OCT;17(5):1592-1601.

(8) Jafari-Nodoushan M, Barzin J, Mobedi H. Size and morphology controlling of PLGA microparticles produced by electro hydrodynamic atomization. Polym Adv Technol 2015 MAY;26(5):502-513.

(9) C. S. Yee. The Development of PVP-based Solid Dispersions using Hot Melt Extrusion for the Preparation of Immediate Release Formulations University of East Anglia; 2013.

(10) Zhang L, Huang J, Si T, Xu RX. Coaxial electrospray of microparticles and nanoparticles for biomedical applications. Expert Rev Med Devices 2012 NOV;9(6):595-612. 
(11) Haj-Ahmad R, Rasekh M, Nazari K, Li Y, Fu Y, Li B, et al. EHDA Spraying: A MultiMaterial Nano-Engineering Route. Curr Pharm Des 2015;21(22):3239-3247.

(12) Zhang C, Chang M, Ahmad Z, Hu W, Zhao D, Li J. Stable single device multi-pore electrospraying of polymeric microparticles via controlled electrostatic interactions. Rsc Advances 2015;5(107):87919-87923.

(13) Disintegration of water drops in an electric field. Proceedings of the Royal Society of London A: Mathematical, Physical and Engineering Sciences: The Royal Society; 1964.

(14) Electrically driven jets. Proceedings of the Royal Society of London A: Mathematical, Physical and Engineering Sciences: The Royal Society; 1969.

(15) Reneker DH, Yarin AL. Electrospinning jets and polymer nanofibers. Polymer 2008;49(10):2387-2425.

(16) Hohman MM, Shin M, Rutledge G, Brenner MP. Electrospinning and electrically forced jets. I. Stability theory. Phys Fluids 2001;13(8):2201-2220.

(17) Yu, D. Williams, G. , Wang, X. , Yang, J. , Li, X. , Qian, W. and Li, Y. Polymer-based nanoparticulate solid dispersions prepared by a modified electrospraying process. Journal of Biomedical Science and Engineering 2011;4:741-749.

(18) Bohr A, Kristensen J, Dyas M, Edirisinghe M, Stride E. Release profile and characteristics of electrosprayed particles for oral delivery of a practically insoluble drug. J R Soc Interface 2012 Oct 7;9(75):2437-2449.

(19) Jahangiri A, Barzegar-Jalali M, Garjani A, Javadzadeh Y, Hamishehkar H, Rameshrad M, et al. Physicochemical characterization and pharmacological evaluation of ezetimibe-PVP K30 solid dispersions in hyperlipidemic rats. Colloids and Surfaces B: Biointerfaces 2015 10/1;134:423-430.

(20) Leuner C, Dressman J. Improving drug solubility for oral delivery using solid dispersions. European Journal of Pharmaceutics and Biopharmaceutics 2000 7/3;50(1):47-60. 
(21) Illangakoon UE, Gill H, Shearman GC, Parhizkar M, Mahalingam S, Chatterton NP, et al. Fast dissolving paracetamol/caffeine nanofibers prepared by electrospinning. Int J Pharm 2014 12/30;477(1-2):369-379.

(22) Adibkia K, Barzegar-Jalali M, Maheri-Esfanjani H, Ghanbarzadeh S, Shokri J, Sabzevari A, et al. Physicochemical characterization of naproxen solid dispersions prepared via spray drying technology. Powder Technol 2013 9;246:448-455.

(23) Payab S, Davaran S, Tanhaei A, Fayyazi B, Jahangiri A, Farzaneh A, et al. Triamcinolone acetonide-Eudragit (R) RS100 nanofibers and nanobeads: Morphological and physicochemical characterization. Artif Cell Nanomed Biotechnol 2016 JAN 2;44(1):362- 369.

(24) Nazari K, Kontogiannidou E, Ahmad RH, Gratsani A, Rasekh M, Arshad MS, et al. Development and characterisation of cellulose based electrospun mats for buccal delivery of non-steroidal anti-inflammatory drug (NSAID). European Journal of Pharmaceutical Sciences 2017 5/1;102:147-155.

(25) Sethia S, Squillante E. Solid dispersion of carbamazepine in PVP K30 by conventional solvent evaporation and supercritical methods. Int J Pharm 2004 3/19;272(1-2):1-10.

(26) Van den Mooter G, Wuyts M, Blaton N, Busson R, Grobet P, Augustijns P, et al. Physical stabilisation of amorphous ketoconazole in solid dispersions with polyvinylpyrrolidone K25. European Journal of Pharmaceutical Sciences 2001 1;12(3):261- 269.

(27) Hancock BC, Carlson GT, Ladipo DD, Langdon BA, Mullarney MP. Comparison of the mechanical properties of the crystalline and amorphous forms of a drug substance. Int J Pharm 2002 7/8;241(1):73-85.

(28) Hancock BC, Zografi G. Characteristics and Significance of the Amorphous State in Pharmaceutical Systems. Journal of Pharmaceutical Sciences 1997 January 1997;86(1):1-12.

(29) ELAMIN A, AHLNECK C, ALDERBORN G, NYSTROM C. Increased Metastable Solubility of Milled Griseofulvin, Depending on the Formation of a Disordered SurfaceStructure. Int J Pharm 1994 OCT 20;111(2):159-170. 
(30) Hancock B, Parks M. What is the true solubility advantage for amorphous pharmaceuticals? Pharm Res 2000 APR;17(4):397-404.

(31) Graeser KA, Patterson JE, Rades T. Applying Thermodynamic and Kinetic Parameters to Predict the Physical Stability of Two Differently Prepared amorphous forms of simvastatin.. Current drug delivery 2009;6(4):374-382.

(32) Craig D, Royall P, Kett V, Hopton M. The relevance of the amorphous state to pharmaceutical dosage forms: glassy drugs and freeze dried systems. Int J Pharm 1999 MAR $15 ; 179(2): 179-207$.

(33) Songsurang K, Praphairaksit N, Siraleartmukul K, Muangsin N. Electrospray Fabrication of Doxorubicin-Chitosan-Tripolyphosphate Nanoparticles for Delivery of Doxorubicin. Arch Pharm Res 2011 APR;34(4):583-592.

(34) Manna L, Banchero M, Sola D, Ferri A, Ronchetti S, Sicardi S. Impregnation of PVP microparticles with ketoprofen in the presence of supercritical CO2. The Journal of Supercritical Fluids 2007 10;42(3):378-384.

(35) Mura P, Moyano J, Gonzalez-Rodriguez M, Rabasco-Alvarez A, Cirri M, Maestrelli F. Characterization and dissolution properties of ketoprofen in binary and ternary solid dispersions with polyethylene glycol and surfactants. Drug Dev Ind Pharm 2005;31(4-5):425- 434.

(36) Kazarian SG, Martirosyan GG. Spectroscopy of polymer/drug formulations processed with supercritical fluids: in situ ATR-IR and Raman study of impregnation of ibuprofen into PVP. Int J Pharm 2002 1/31;232(1-2):81-90.

(37) Di Martino P, Joiris E, Gobetto R, Masic A, Palmieri GF, Martelli S. Ketoprofenpoly(vinylpyrrolidone) physical interaction. J Cryst Growth 2004 4/15;265(1-2):302-308. 
Tables and Figures

Figures

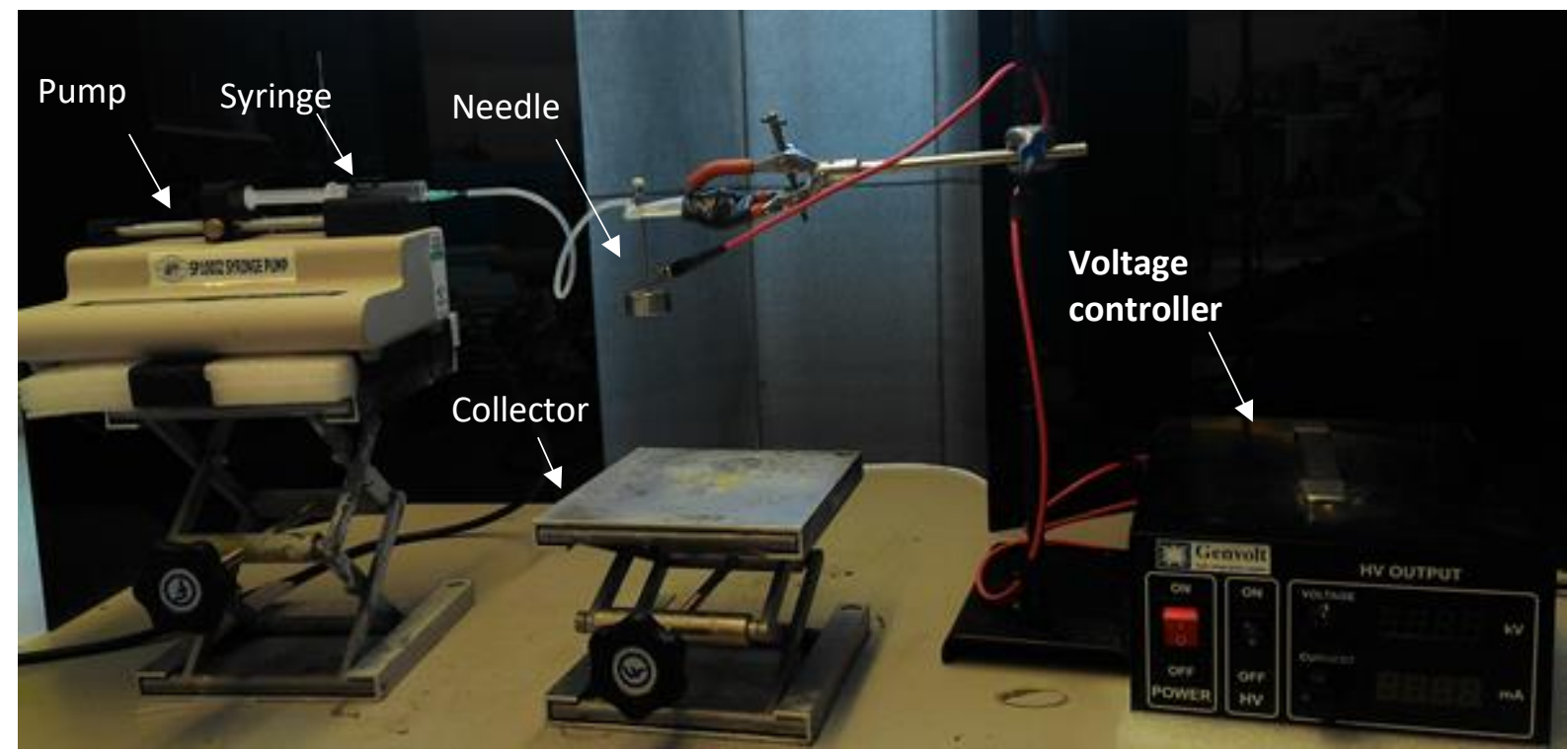

Figure 1 

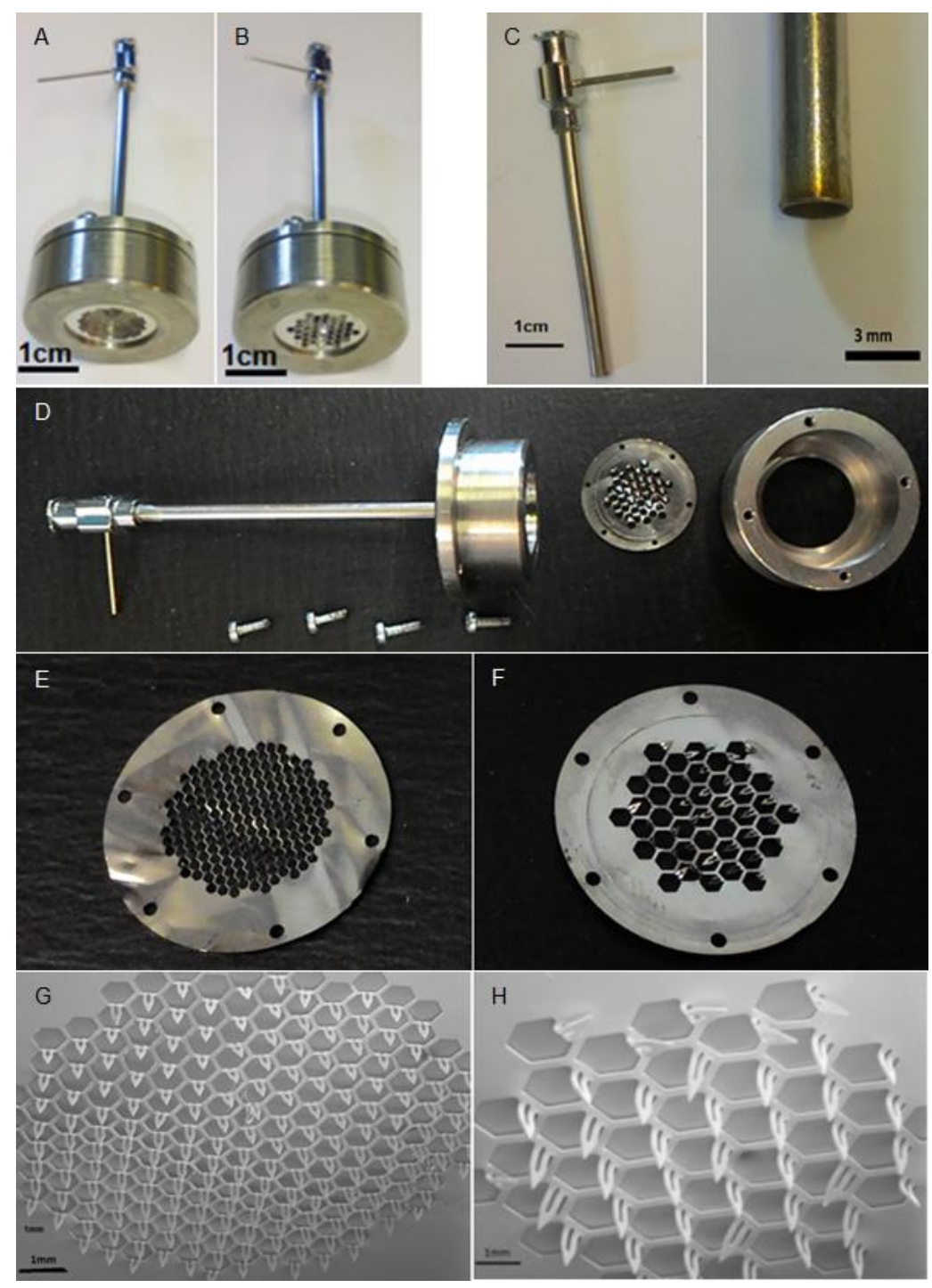

Figure 2 


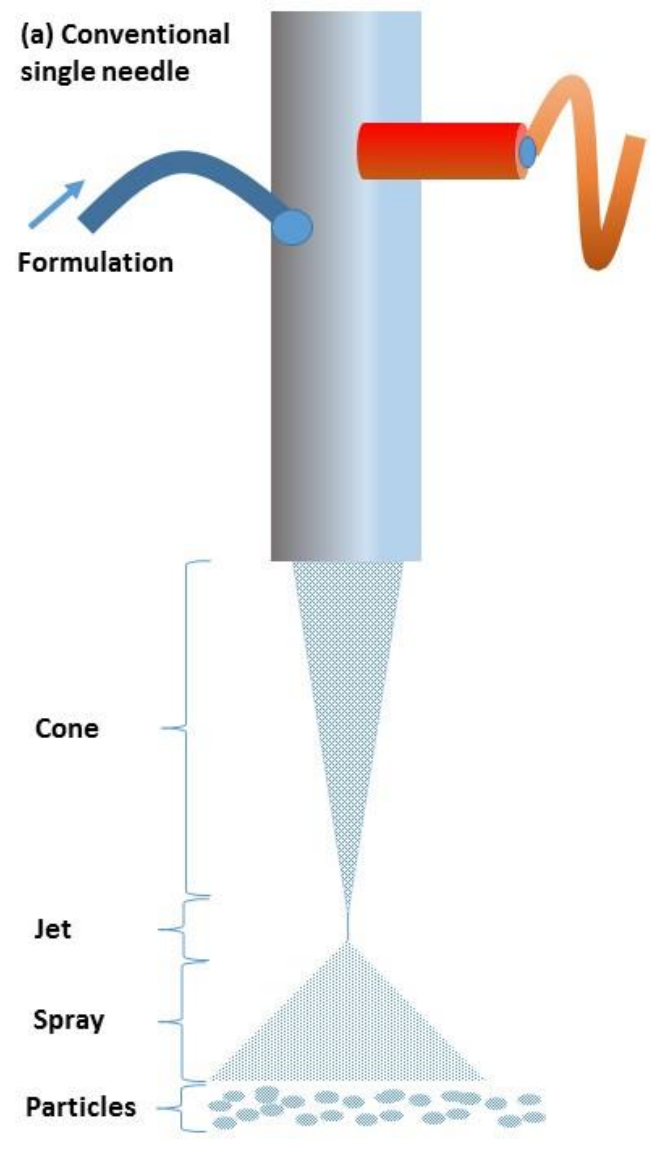

(b) Multi-nozzle atomisers

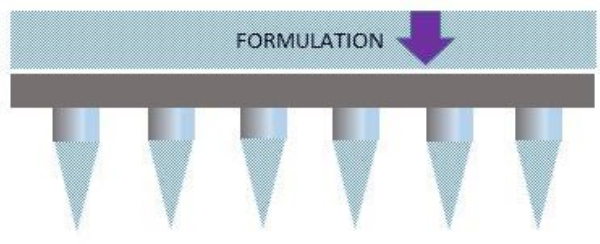

(c) Pore based atomisers



(d) Multi-Tip Emitter atomiser

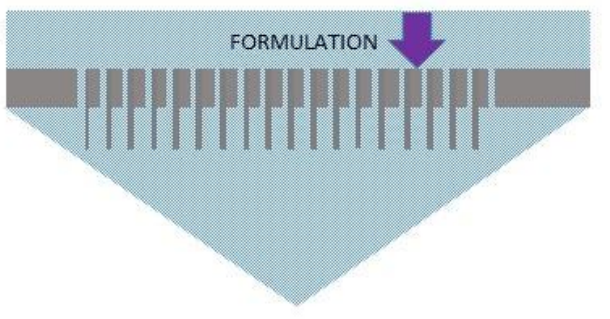

Figure 3 


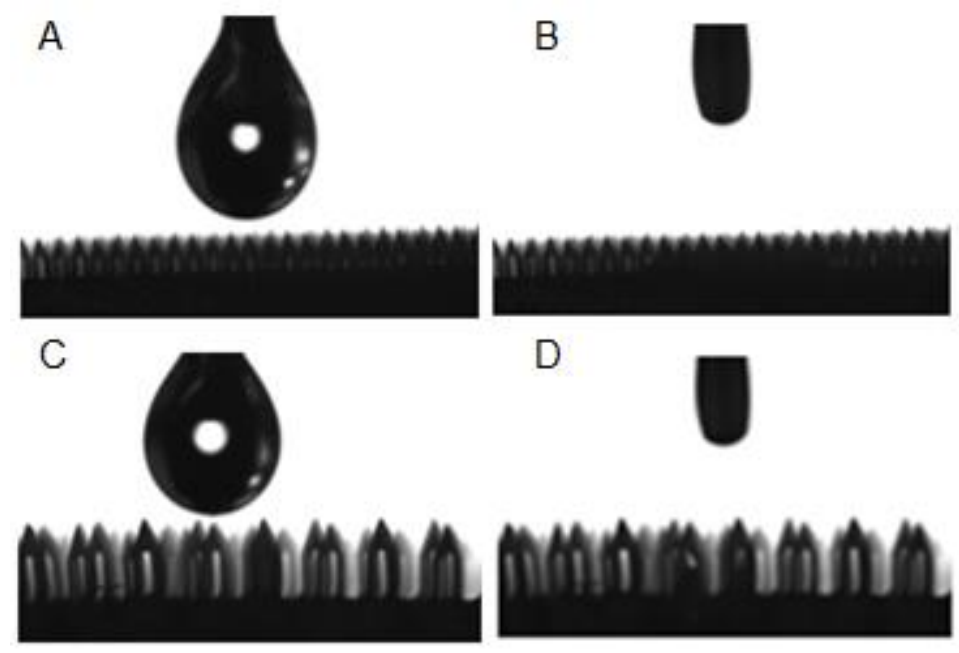

Figure 4 


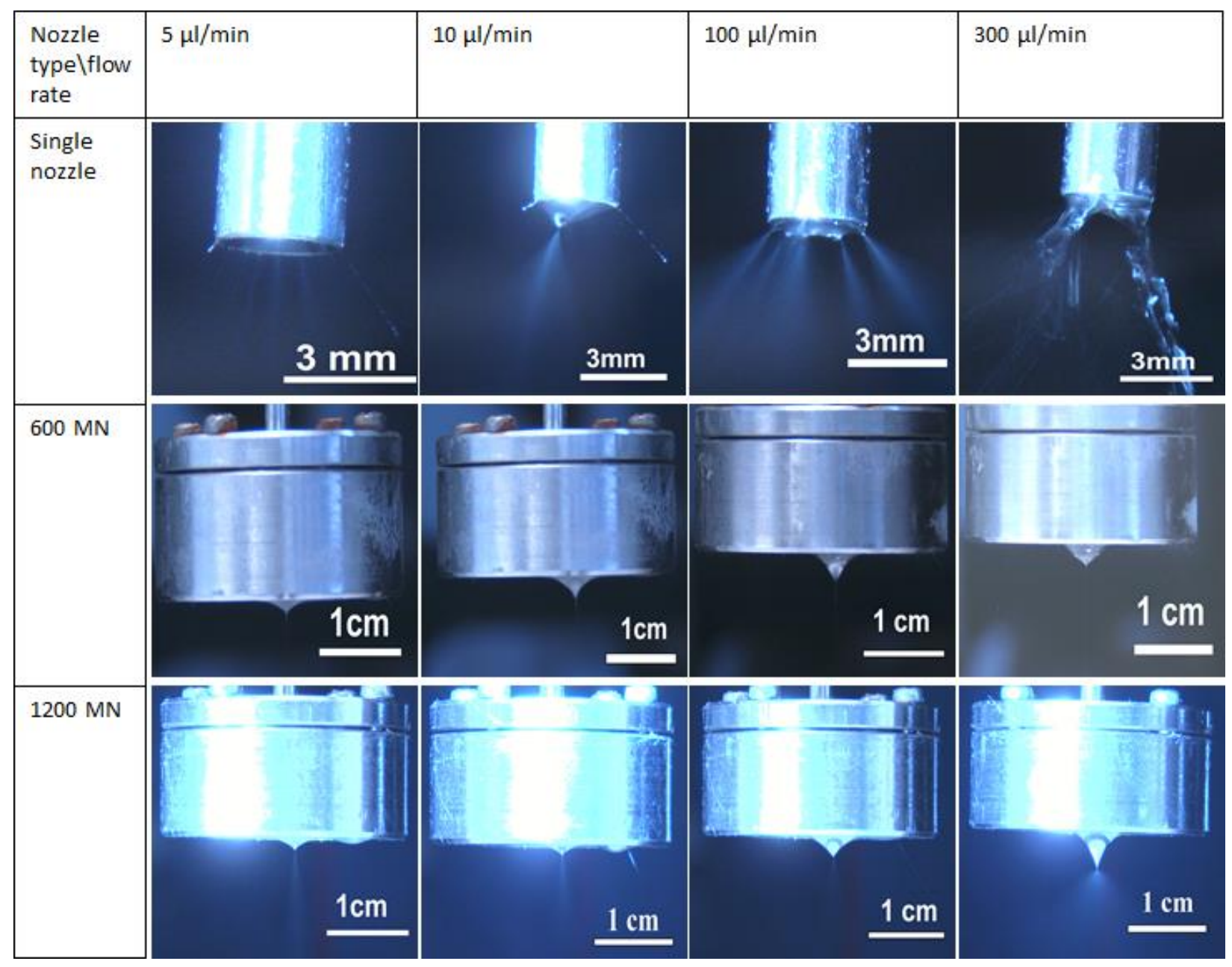

Figure 5 


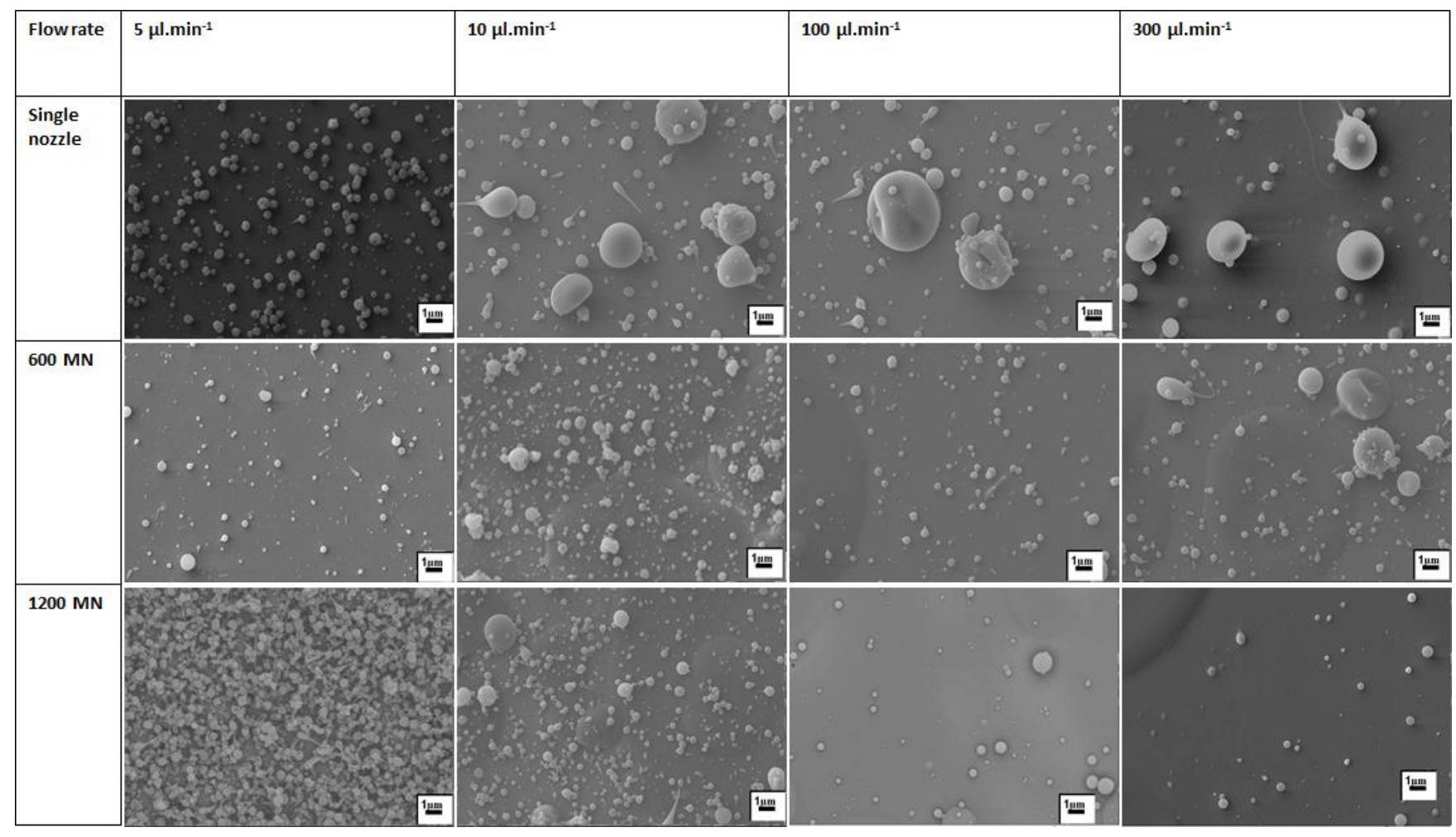

Figure 6 

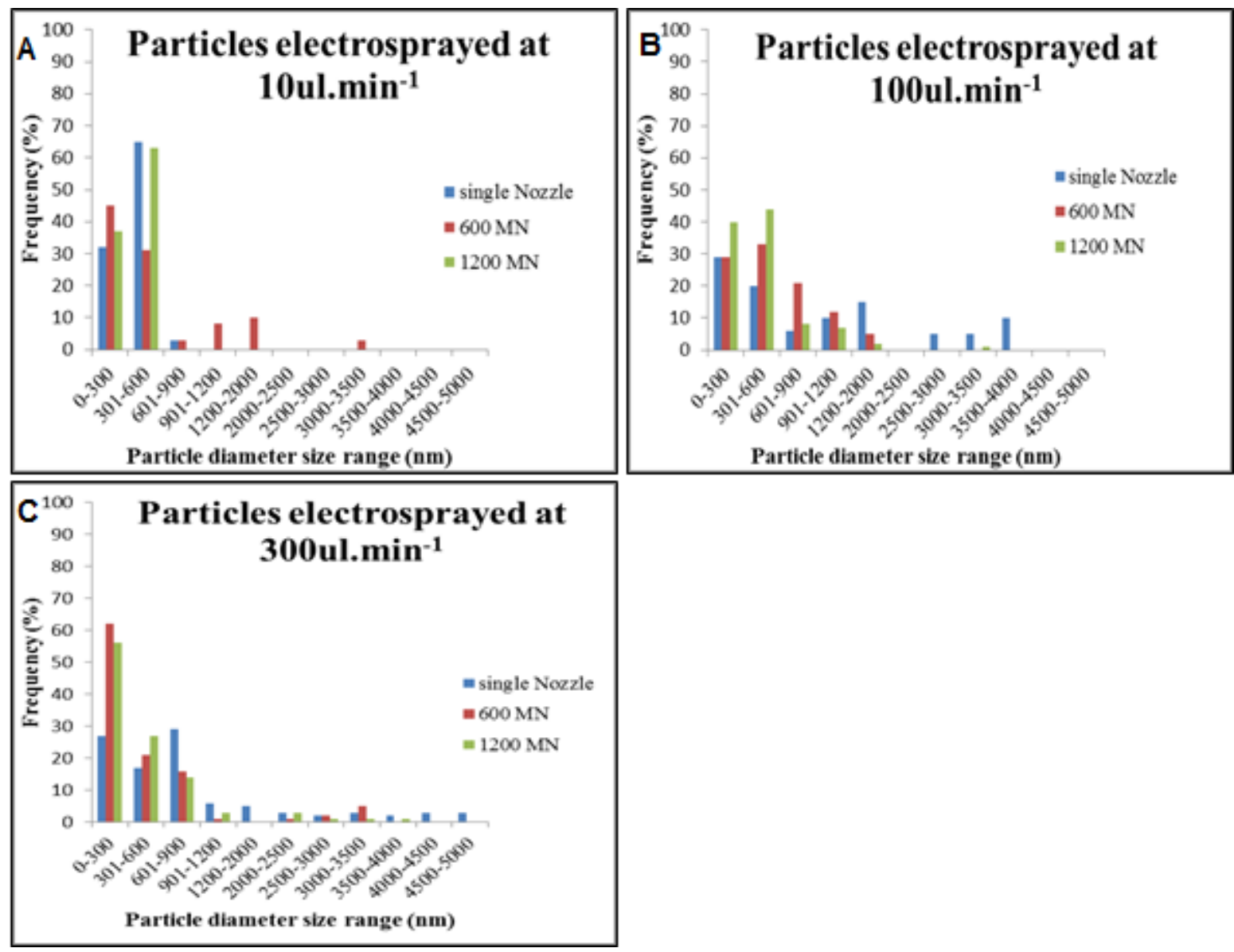

Figure 7 

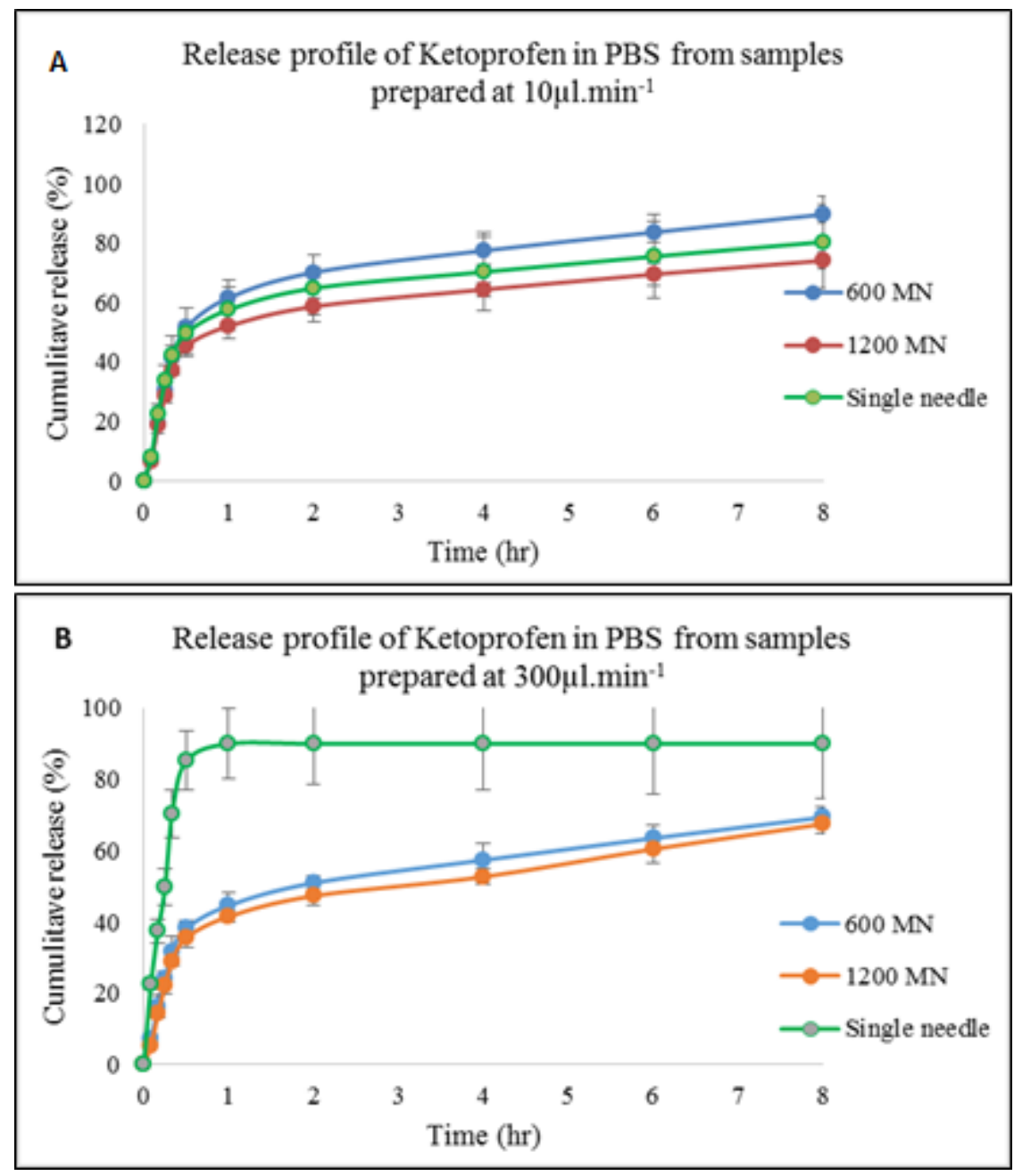

Figure 8 

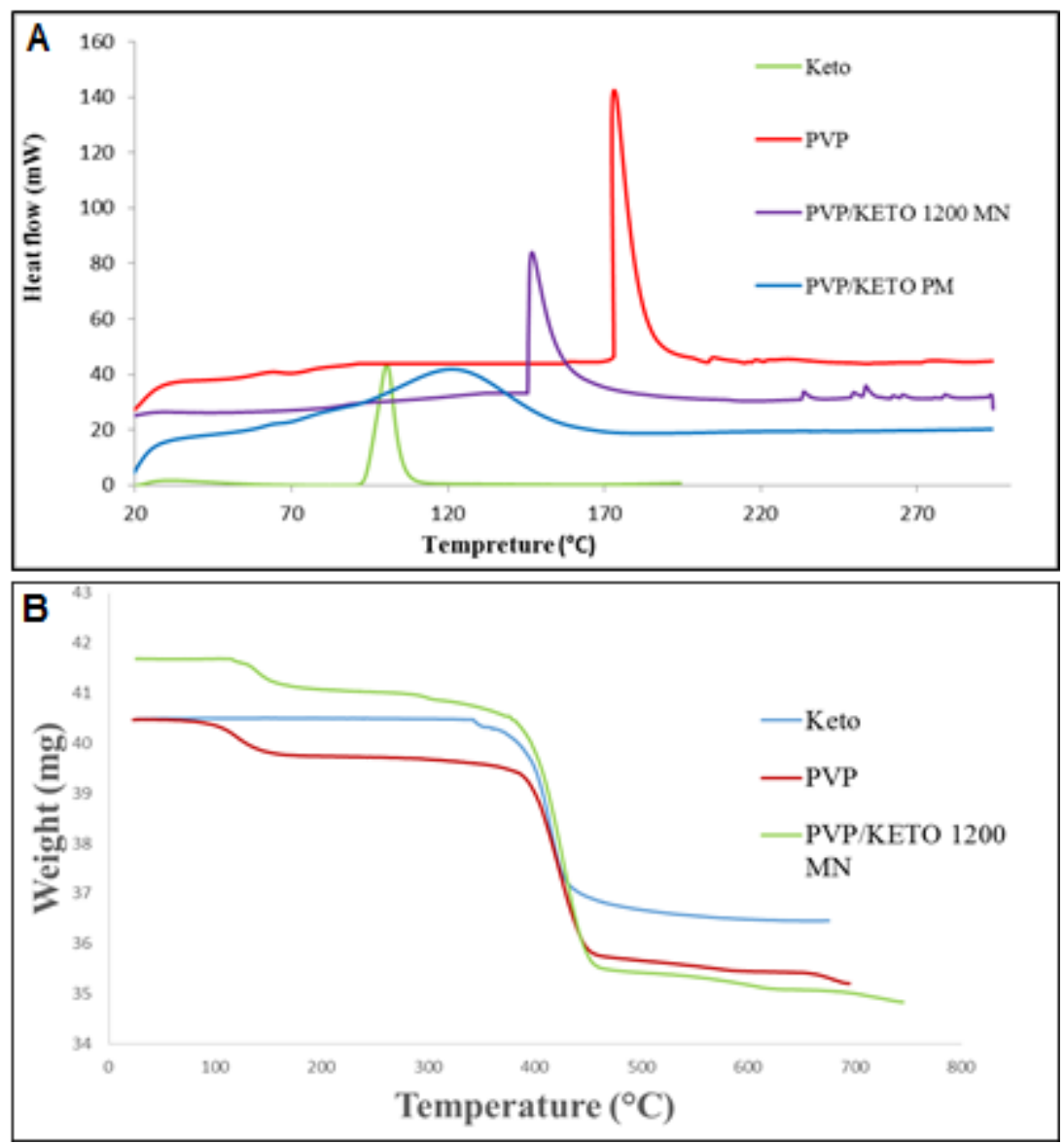

Figure 9 

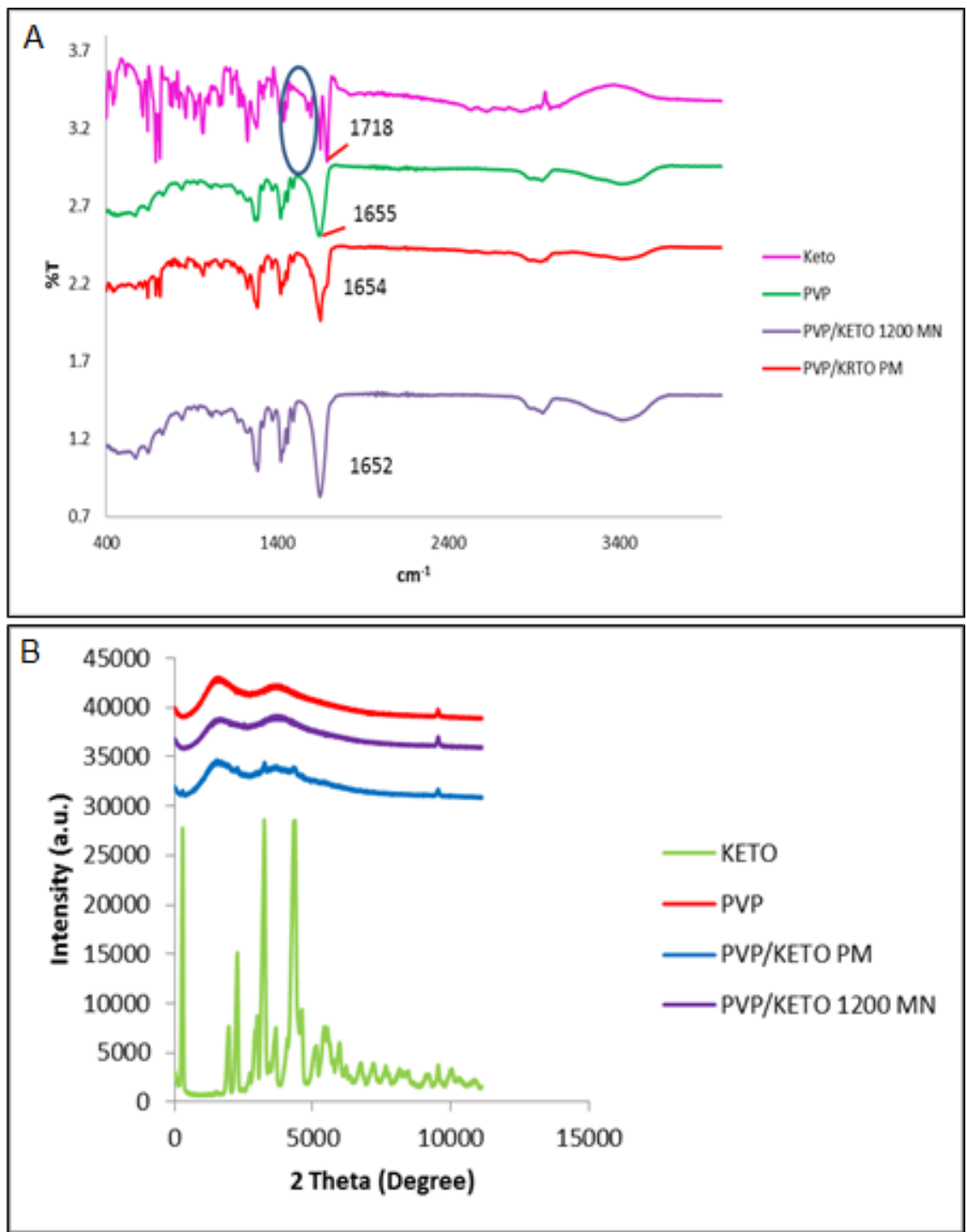

Figure 10 
Tables

Table 1.

\begin{tabular}{|l|l|l|l|l|}
\hline Solution & $\begin{array}{l}\text { Viscosity } \\
(\mathrm{m} . P a s)\end{array}$ & $\begin{array}{l}\text { Electric } \\
\text { conductivity } \\
(\mu \mathrm{S} . \mathrm{cm})\end{array}$ & Density $\left(\mathrm{Kg} / \mathrm{m}^{3}\right)$ & $\begin{array}{l}\text { Surface tension } \\
(\mathrm{mN} / \mathrm{m})\end{array}$ \\
\hline Ethanol & $0.97 \pm 0.01$ & $0.127 \pm 0.02$ & $19.4096 \pm 0.12$ & $0.035 \pm 0.001$ \\
\hline $\begin{array}{l}(80: 20 \mathrm{v} / \mathrm{v}) \\
\text { Ethanol: } \mathrm{H}_{2} \mathrm{O}\end{array}$ & $1.55 \pm 0.02$ & $0.605 \pm 0.01$ & $20.9981 \pm 0.09$ & $0.032 \pm 0.002$ \\
\hline $\begin{array}{l}(1 \% \mathrm{w} / \mathrm{v}) \mathrm{KETO} \\
\text { in }(80: 20 \mathrm{v} / \mathrm{v}) \\
\text { Ethanol: } \mathrm{H}_{2} \mathrm{O}\end{array}$ & $1.57 \pm 0.01$ & $6.29 \pm 0.03$ & $20.9492 \pm 0.05$ & $0.033 \pm 0.002$ \\
\hline $\begin{array}{l}(5 \% \mathrm{w} / \mathrm{v}) \mathrm{PVP} \text { in } \\
(80: 20 \mathrm{v} / \mathrm{v})\end{array}$ & $1.58 \pm 0.02$ & $10.15 \pm 0.01$ & $19.6624 \pm 0.14$ & $0.037 \pm 0.002$ \\
Ethanol: $\mathrm{H}_{2} \mathrm{O}$ & & $16.54 \pm 0.03$ & $20.6029 \pm 0.01$ & $0.038 \pm 0.001$ \\
\hline $\begin{array}{l}* P V P \& \mathrm{KETO} \\
\text { in }(80: 20 \mathrm{v} / \mathrm{v}) \\
\text { Ethanol: } \mathrm{H}_{2} \mathrm{O}\end{array}$ & $3.24 \pm 0.01$ & & & \\
\hline
\end{tabular}

*The concentration of PVP is 5\% w/v and KETO is $5 \% \mathrm{w} / \mathrm{v}$ of the $5 \% \mathrm{w} / \mathrm{v}$ of PVP.

Table 2.

\begin{tabular}{|l|l|l|l|l|l|l|}
\hline MN & Flow rate & Cone & Cone & Cone & Jet $(\mathrm{cm})$ & Spray width $(\mathrm{SW})(\mathrm{cm})$ \\
& $\left(\mu 1 . \mathrm{min}^{-1}\right)$ & $\begin{array}{l}\text { shape } \\
(\mathrm{CS})\end{array}$ & $\begin{array}{l}\text { diameter } \\
(\mathrm{d})(\mathrm{cm})\end{array}$ & $\begin{array}{l}\mathrm{L})(\mathrm{cm}) \\
\text { length }\end{array}$ & & \\
\hline 600 & 100 & skewed & 0.6 & 0.3 & 1.2 & 0.2 \\
\cline { 2 - 7 } & 300 & inverted & 0.6 & 0.2 & 1.1 & 0.1 \\
\hline 1200 & 100 & skewed & 0.7 & 0.3 & 0.04 & 0.6 \\
\cline { 2 - 8 } & 300 & inverted & 0.6 & 0.3 & 0.06 & 1.5 \\
\hline
\end{tabular}




\section{Table 3}

\begin{tabular}{|l|l|l|}
\hline Flow rate & Nozzle configuration & Encapsulation efficiency (\%) \\
\hline \multirow{3}{*}{$10 \mu 1 . \mathrm{min}^{-1}$} & Single needle & $85.23 \pm 0.3$ \\
\cline { 2 - 3 } & $600 \mathrm{MN}$ & $79.63 \pm 0.2$ \\
\cline { 2 - 3 } & $1200 \mathrm{MN}$ & $87.82 \pm 0.3$ \\
\hline \multirow{3}{*}{$300 \mu 1 . \mathrm{min}^{-1}$} & Single needle & $32.14 \pm 5.3$ \\
\cline { 2 - 3 } & $600 \mathrm{MN}$ & $55.34 \pm 1.5$ \\
\cline { 2 - 3 } & $1200 \mathrm{MN}$ & $72.62 \pm 1.2$ \\
\hline
\end{tabular}

